\title{
Cohomological approach to the graded Berezinian
}

\author{
Tiffany Covolo
}

\begin{abstract}
We develop the theory of linear algebra over a $\left(\mathbb{Z}_{2}\right)^{n}$-commutative algebra $(n \in \mathbb{N})$, which includes the well-known super linear algebra as a special case $(n=1)$. Examples of such graded-commutative algebras are the Clifford algebras, in particular the quaternion algebra $\mathbb{H}$. Following a cohomological approach, we introduce analogues of the notions of trace and determinant. Our construction reduces in the classical commutative case to the coordinate-free description of the determinant by means of the action of invertible matrices on the top exterior power, and in the supercommutative case it coincides with the well-known cohomological interpretation of the Berezinian.
\end{abstract}

Mathematics Subject Classification (2010). 16W50, 17A70, 11R52, 15A15, 15A66, 16E40. Keywords. Graded linear algebra, graded trace and Berezinian, quaternions, Clifford algebra.

\section{Introduction}

Remarkable series of algebras, such as the algebra of quaternions and, more generally, Clifford algebras turn out to be graded-commutative. Originated in [1] and [2], this idea was developed in [10] and [11]. The grading group in this case is $\left(\mathbb{Z}_{2}\right)^{n+1}$, where $n$ is the number of generators, and the graded-commutativity reads as

$$
a b=(-1)^{\langle\tilde{a}, \tilde{b}\rangle} b a
$$

where $\tilde{a}, \tilde{b} \in\left(\mathbb{Z}_{2}\right)^{n+1}$ denote the degrees of the respective homogeneous elements $a$ and $b$, and $\langle\rangle:,\left(\mathbb{Z}_{2}\right)^{n+1} \times\left(\mathbb{Z}_{2}\right)^{n+1} \rightarrow \mathbb{Z}_{2}$ is the standard scalar product of binary $(n+1)$-vectors (see Section 2$)$. This choice of the graded-commutativity has various motivations. First, it is the intuitive extension of the well-known superalgebra, which corresponds to this $\left(\mathbb{Z}_{2}\right)^{n}$-commutativity for $n=1,\langle$, being in this case just classical multiplication. Secondly, it was proved in [11] that such $\left(\mathbb{Z}_{2}\right)^{n}$-commutativity is universal among graded-commutative algebras. That is, if $\Gamma$ is a finitely generated Abelian group, then for an arbitrary $\Gamma$-gradedcommutative algebra $A$ with graded-commutativity of the form $a b=(-1)^{\beta(\tilde{a}, \tilde{b})} b a$, with $\beta: \Gamma \times \Gamma \rightarrow \mathbb{Z}_{2}$ a bilinear symmetric map, it exists $n \in \mathbb{N}$ such that $A$ is $\left(\mathbb{Z}_{2}\right)^{n}$-commutative (in the sense of $(1.1)$ ). 
First steps towards the $\left(\mathbb{Z}_{2}\right)^{n}$-graded version of linear algebra were done in [6]. The notion of graded trace for all endomorphisms and that of graded Berezinian for 0-degree automorphisms were introduced in the most general framework of an arbitrary free module (of finite rank) over a $\left(\mathbb{Z}_{2}\right)^{n}$-commutative algebra.

In this paper, we develop a cohomological approach to the notion of graded Berezinian and graded trace. In the super case, this approach is originally due to O. V. Ogievetskii and I. B. Penkov ([12]), but we will mostly refer to the description given in [9]. Similarly to this latter, we define a graded analogue of the Koszul complex and the graded Berezinian module associated to a given free module of finite rank. We believe this to be the first step towards the conception of a generalization of the Berezinian integral over a multi-graded (i.e. $\left(\mathbb{Z}_{2}\right)^{n}$-graded) manifold, see [3] and [4] for the definition and a first study of this latter.

The paper is organized as follows. We recall the basic notions of graded linear algebra in Section 2 and derive the graded matrix calculus in Section 3. In Section 4, we present our first main result, a cohomological interpretation of the graded Berezinian. In Section 5, we give a similar description of the graded trace. It is worth noticing that the cohomological description of the graded trace of arbitrary even matrices leads to interesting restrictions for the grading group $\left(\mathbb{Z}_{2}\right)^{n}$, namely $n$ has to be odd. Furthermore, the parity changing operator has to be chosen in a canonical way and corresponds to the element $(1,1, \ldots, 1)$ of $\left(\mathbb{Z}_{2}\right)^{n}$.

We have to note that there is an alternative approach to the generalization of superalgebras and related notions, which makes use of category theory. This approach follows from results by Scheunert in [13] (in the Lie algebras setting) and Nekludova (in the commutative algebra setting). An explicit description of the results of the latter can be found in [8]. This other method to treat the problem and its consequences in the $\left(\mathbb{Z}_{2}\right)^{n}$-commutative case, will be the object of a separate work [5].

Acknowledgements. The author is pleased to thank Norbert Poncin whose suggestions initiate the paper, Jean-Philippe Michel for enlightening discussions and Dimitry Leites who made her aware of Nekludova's work. The author is grateful to Valentin Ovsienko for the contributions he gave through the development and the finalization of the paper.

The author thanks the Luxembourgian NRF for support via AFR PhD grant 2010$1,786207$.

\section{Graded linear algebra}

In this section, we give a brief survey of the main notions of linear algebra over graded-commutative algebras. 
Consider an Abelian group $(\Gamma,+)$ endowed with a symmetric bi-additive map

$$
\langle,\rangle: \Gamma \times \Gamma \rightarrow \mathbb{Z}_{2} .
$$

We call $\Gamma$ the grading group. This group admits a natural splitting $\Gamma=\Gamma_{\overline{0}} \cup \Gamma_{\overline{1}}$, where $\Gamma_{\overline{0}}$ is the subgroup characterized by $\langle\gamma, \gamma\rangle=0$ for all $\gamma \in \Gamma_{\overline{0}}$, and where $\Gamma_{\overline{1}}$ is the set characterized by $\langle\gamma, \gamma\rangle=1$ for all $\gamma \in \Gamma_{\overline{1}}$. We call $\Gamma_{\overline{0}}$ and $\Gamma_{\overline{1}}$ the even subgroup and odd part, respectively.

In this paper, we restrict the considerations to the case $\Gamma=\left(\mathbb{Z}_{2}\right)^{n}$, for some fixed $n \in \mathbb{N}$, equipped with the standard scalar product

$$
\langle x, y\rangle=\sum_{1 \leq i \leq n} x_{i} y_{i}
$$

of $n$-vectors, defined over $\mathbb{Z}_{2}$. Our main example are the Clifford algebras equipped with the grading described in Example 2.1 (see next section).

2.1. Graded-commutative algebras. A graded vector space is a direct sum

$$
V=\bigoplus_{\gamma \in \Gamma} V^{\gamma}
$$

of vector spaces $V^{\gamma}$ over a commutative field $\mathbb{K}$ (that we always assume of characteristic 0). An endomorphism of $V$ is a $\mathbb{K}$-linear map from $V$ to $V$ that preserves the degree; we denote by $\operatorname{End}_{\mathbb{K}}(V)$ the space of endomorphisms.

A $\Gamma$-graded algebra is an algebra $A$ which has a structure of a $\Gamma$-graded vector space $A=\bigoplus_{\gamma \in \Gamma} A^{\gamma}$ such that the operation of multiplication respects the grading for all $\alpha, \beta \in \Gamma$,

$$
A^{\alpha} A^{\beta} \subset A^{\alpha+\beta} .
$$

We always assume $A$ associative and unital.

An element $a \in A^{\gamma}$ is called homogeneous of degree $\gamma$. For every homogeneous element $a$, we denote by $\tilde{a}$ its degree. Because of the even-odd splitting of the grading group, one also has

$$
A=A_{\overline{0}} \oplus A_{\overline{1}},
$$

where $a \in A_{\overline{0}}$ or $A_{\overline{1}}$ if $\tilde{a}$ is even or odd, respectively. For simplicity, in most formulas below the involved elements are assumed to be homogeneous. These expressions are then extended to arbitrary elements by linearity.

A $\Gamma$-graded algebra is called $\Gamma$-commutative if

$$
a b=(-1)^{\langle\tilde{a}, \tilde{b}\rangle} b a .
$$

In particular, every odd element squares to zero. 
Example 2.1. As we have mentioned in the Introduction, a Clifford algebra $\mathrm{Cl}_{n}$ of $n$ generators (over $\mathbb{R}$ or $\mathbb{C}$ ) is a $\left(\mathbb{Z}_{2}\right)^{n+1}$-commutative algebra ([11]). The grading is given on the generators $e_{i}$ of $\mathrm{Cl}_{n}$ as follows

$$
\tilde{e}_{i}=(0, \ldots, 0,1,0, \ldots 0,1) \in\left(\mathbb{Z}_{2}\right)^{n+1}
$$

where 1 is at the $i$-th and at the last position.

2.2. Graded modules. A graded left module $M$ over a $\Gamma$-commutative algebra $A$ is a left $A$-module with a $\Gamma$-graded vector space structure $M=\bigoplus_{\gamma \in \Gamma} M^{\gamma}$ such that the $A$-module structure respects the grading, i.e. for all $\alpha, \beta \in \Gamma$,

$$
A^{\alpha} M^{\beta} \subset M^{\alpha+\beta} \text {. }
$$

The notion of graded right module over $A$ is defined analogously. Thanks to the graded-commutativity of $A$, a left $A$-module structure induces a compatible right $A$-module structure given by

$$
m a:=(-1)^{\langle\tilde{a}, \tilde{m}\rangle} a m,
$$

and vice-versa ${ }^{1}$. Hence, we identify the two concepts. An $A$-module is called free of total rank $r \in \mathbb{N}$ if it admits a basis of $r$ homogeneous elements $\left\{e_{s}\right\}_{s=1, \ldots, r}$. In this case, every element $m \in M$ can be decomposed in a basis either with left or right coefficients, which are clearly related through (2.2).

A morphism of $A$-modules, is a map $\ell: M \rightarrow N$ which is $A$-linear of degree zero (i.e. $\left.\ell\left(M^{\alpha}\right) \subset N^{\alpha}, \forall \alpha \in \Gamma\right)$. We will denote the set of such maps by $\operatorname{Hom}_{A}(M, N)$. We usually refer to this set as the categorical Hom since $A$-modules with these degree-preserving $A$-linear maps form a category $\mathrm{Gr}_{\Gamma} \operatorname{Mod}_{A}$.

We remark that the Hom set of graded $A$-modules is not a graded $A$-module itself. The internal Hom of the category $\mathrm{Gr}_{\Gamma} \operatorname{Mod}_{A}$ is

$$
\operatorname{Hom}_{A}(M, N):=\bigoplus_{\gamma \in \Gamma} \operatorname{Hom}_{A}^{\gamma}(M, N),
$$

where each $\operatorname{Hom}_{A}^{\gamma}(M, N)$ consists of $A$-linear maps $\ell: M \rightarrow N$ of degree $\gamma$, that is additive maps satisfying

$$
\begin{gathered}
\ell(a m)=(-1)^{\langle\gamma, \tilde{a}\rangle} a \ell(m) \quad(\text { or equivalently, } \ell(m a)=\ell(m) a) \\
\text { and } \quad \ell\left(M^{\alpha}\right) \subset N^{\alpha+\gamma} .
\end{gathered}
$$

The $A$-module structure of $\operatorname{Hom}_{A}(M, N)$ is given by

$$
(a \cdot \ell)(-):=(-1)^{\langle\tilde{\ell}, \tilde{a}\rangle} \ell(a \cdot-) \quad(\text { or equivalently, }(\ell \cdot a)(-)=\ell(a \cdot-)) .
$$

\footnotetext{
${ }^{1}$ We have to note that, even though the most natural, (2.2) is not the only possible choice. In general, for any fixed $\gamma \in \Gamma$, setting $m a:=(-1)^{\langle\tilde{a}, \tilde{m}+\gamma} a m$ also defines a right module structure on a left module. This situation also appears in superalgebra [8].
} 
Remark 2.2. The categorical Hom coincides with the 0-degree part of the internal Hom, i.e. $\operatorname{Hom}_{A}(M, N)=\operatorname{Hom}_{A}^{0}(M, N)$. By abuse of notation, we also refer to the elements of the internal Hom as morphisms. To make clear the distinction between categorical and internal hom, we often add the adjective "graded" in the latter case.

We define graded endomorphisms and graded automorphisms of an $A$-module $M$ by

and

$$
\begin{aligned}
& \operatorname{End}_{A}(M):=\operatorname{Hom}_{A}(M, M) \\
& \operatorname{Aut}_{A}(M):=\left\{\ell \in \operatorname{End}_{A}(M): \ell \text { invertible }\right\},
\end{aligned}
$$

and their degree-preserving analogues by

$$
\operatorname{End}_{A}(M):=\operatorname{End}_{A}^{0}(M) \text { and } \operatorname{Aut}_{A}(M):=\operatorname{Aut}_{A}^{0}(M) .
$$

In situations where it is not misleading, we will drop the subscript and just write $\operatorname{Hom}(M, N), \operatorname{End}(M), \operatorname{Aut}(M)$, etc.

The dual of a graded $A$-module $M$ is the graded $A$-module $M^{*}:=\operatorname{Hom}(M, A)$. As for classical modules, if $M$ is free with basis $\left\{e_{i}\right\}_{i=1, \ldots, r}$ then its dual module $M^{*}$ is also free of same rank. Its basis $\left\{\varepsilon^{i}\right\}_{i=1, \ldots, r}$ is defined as usual by

$$
\varepsilon^{i}\left(e_{j}\right)=\delta_{j}^{i}, \quad \forall i, j
$$

where $\delta_{j}^{i}$ is the Kronecker delta. Note that this implies $\tilde{\varepsilon^{i}}=\tilde{e_{i}}$ for all $i$.

2.3. Lie algebras, derivations. A $\Gamma$-colored Lie algebra $A$ is a $\Gamma$-graded algebra in which the multiplication operation (denoted $[$,$] ) verify the following two$ conditions, for all homogeneous elements $a, b, c \in A$.

1) Graded skew-symmetry:

$$
[a, b]=-(-1)^{\langle\tilde{a}, \tilde{b}\rangle}[b, a] ;
$$

2) Graded Jacoby identity:

$$
[a,[b, c]]=[[a, b], c]+(-1)^{\langle\tilde{a}, \tilde{b}\rangle}[b,[a, c]] .
$$

Natural examples of colored Lie algebras are $\Gamma$-graded associative algebras with the graded commutator

$$
[a, b]=a b-(-1)^{\langle\tilde{a}, \tilde{b}\rangle} b a .
$$

If $A$ is a $\left(\mathbb{Z}_{2}\right)^{n}$-colored Lie algebra, its 0 -degree part $A^{0}$ is a classical Lie algebra. 
An homogeneous derivation of degree $\gamma$ of a $\Gamma$-graded algebra $A$ over a field $\mathbb{K}$, is a $\mathbb{K}$-linear map of degree $\gamma D \in \operatorname{End}_{\mathbb{K}}^{\gamma}(A)$ which verifies the graded Leibniz rule

$$
D(a b)=D(a) b+(-1)^{\langle\tilde{a}, \gamma\rangle} a D(b),
$$

for all homogeneous elements $a, b \in A$.

We denote the set of derivations of degree $\gamma$ of $A$ by $\operatorname{Der}^{\gamma}(A)$. Then, the set of all graded derivations of $A$

$$
\operatorname{Der}(A):=\bigoplus_{\gamma \in \Gamma} \operatorname{Der}^{\gamma}(A)
$$

is a $\Gamma$-graded vector space. It is also an $A$-module, with the $A$-module structure given by $(a D)(x)=a D(x)$. Moreover, considering composition of derivations, $\operatorname{Der}(A)$ is also a colored Lie algebra for the commutator (2.5).

2.4. Graded tensor and symmetric algebras. Let $A$ be a $\Gamma$-commutative algebra.

The tensor product of two graded $A$-modules $M$ and $N$ can be defined as follows. Let us forget, for the moment, the graded structure of $A$, seeing it simply as a noncommutative ring, and consider $M$ and $N$ respectively as right and left modules. In this situation, the notion of tensor product is well-known (see [7]), and the obtained object $M \otimes_{A} N$ is a $\mathbb{Z}$-module. Then, reconsidering the graded structure of the initial objects, we see that $M \otimes_{A} N$ admits an induced $\Gamma$-graded structure

$$
M \otimes_{A} N=\bigoplus_{\gamma \in \Gamma}\left(M \otimes_{A} N\right)^{\gamma}=\bigoplus_{\gamma \in \Gamma} \bigoplus_{\alpha+\beta=\gamma}\left\{\sum m \otimes_{A} n \mid m \in M^{\alpha}, n \in N^{\beta}\right\} .
$$

Moreover, because of the actual two-sided module structure of both $M$ and $N$, the resulting object $M \otimes_{A} N$ have also right and left $A$-module structures, which are by construction compatible (in the sense of (2.2)).

As usual, tensor product of graded $A$-modules can be characterized as a universal object. All classical results and constructions related to the tensor product can then be transferred to the graded case without major difficulties.

Set $M^{\otimes k}=M \otimes_{A} \ldots \otimes_{A} M(n$ factors $M, n \geq 1)$ and $M^{\otimes 0}:=A$. The graded $A$-module

$$
T_{A}^{\bullet} M:=\bigoplus_{k \in \mathbb{N}} M^{\otimes k}
$$

is an associative graded $A$-algebra, called graded tensor algebra, with multiplication

$$
\otimes_{A}: M^{\otimes r} \times M^{\otimes s} \rightarrow M^{\otimes r} \otimes_{A} M^{\otimes s} \simeq M^{\otimes(r+s)} .
$$

The graded $A$-algebra $T_{A}^{\bullet} M$ is in fact bi-graded: it has the classical $\mathbb{N}$-grading (given by the number of factors in $M$ ) that we call weight, and an induced $\Gamma$-grading (see (2.6)) called degree. 

form

Taking the quotient of $T_{A}^{\bullet} M$ by the ideal $J_{S}$ generated by the elements of the

$$
m \otimes m^{\prime}-(-1)^{\left\langle\tilde{m}, \tilde{m^{\prime}}\right\rangle} m^{\prime} \otimes m, \quad \forall m, m^{\prime} \in M
$$

we obtain a $\Gamma$-commutative $A$-algebra $S_{A}^{\bullet}(M)$ called the graded symmetric algebra.

As their classical analogues, both these notions satisfy universal properties.

2.5. Change of parity functors. Unlike the classical super case (i.e. $\Gamma=\mathbb{Z}_{2}$ ), in general we have many different parity reversion functors.

For every $\pi \in \Gamma_{\overline{1}}$, we specify an endofunctor of the category of modules over a $\Gamma$-commutative algebra $A$

$$
\begin{array}{cccc}
\Pi: & \longrightarrow & \operatorname{Gr}_{\Gamma} \operatorname{Mod}_{A} \\
M & \operatorname{Mod}_{A} & \mapsto & \Pi M \\
\operatorname{Hom}_{A}(M, N) \ni f & \mapsto & f^{\Pi} \in \operatorname{Hom}_{A}(\Pi M, \Pi N)
\end{array}
$$

The object $\Pi M$ is defined by

$$
(\Pi M)^{\alpha}:=M^{\alpha+\pi},
$$

for all $\alpha \in \Gamma$, and graded $A$-module structure

$$
\Pi\left(m+m^{\prime}\right):=\Pi m+\Pi m^{\prime} \quad \text { and } \quad \Pi(a m):=(-1)^{\langle\pi, \tilde{a}\rangle} a \Pi m .
$$

The morphism $f^{\Pi} \in \operatorname{Hom}_{A}(\Pi M, \Pi N)$ is defined by

$$
f^{\Pi}(\Pi m):=\Pi(f(m)) .
$$

Clearly, the map $\Pi$ which sends an $A$-module $M$ to the $A$-module $\Pi M$ is an $A$-linear map of degree $\pi$, i.e. $\Pi \in \operatorname{Hom}^{\pi}(M, \Pi M)$.

\section{Graded matrix calculus}

A graded morphism $t: M \rightarrow N$ of free $A$-modules of total rank $r$ and $s$ respectively, can be represented by a matrix over $A$. Fixing a basis $\left\{e_{i}\right\}_{i=1, \ldots, r}$ of $M$ and $\left\{h_{j}\right\}_{j=1, \ldots, s}$ of $N$ and considering elements of the modules as column vectors of right coordinates

$$
M \ni m=\sum e_{j} a^{j} \simeq \mathbf{m}=\left(\begin{array}{c}
a^{1} \\
a^{2} \\
\vdots
\end{array}\right),
$$

the graded morphism is defined by the images of the basis vectors

$$
t(m)=\sum_{j} t\left(e_{i}\right) a^{i}=\sum_{i, j} h_{j} t_{i}^{j} a^{i} .
$$


Hence, applying $t$ corresponds to left multiplication by the matrix $T=\left(t^{j}{ }_{i}\right) \in$ $\mathrm{M}(s \times r ; A)$,

$$
t(m) \simeq T \mathbf{m} .
$$

We have a similar description when considering elements of the modules as row vectors of left coordinates. In this paper, we choose the first approach. This choice is justified by the fact that the graded morphisms are easier to handle when one consider the right module structure, see (2.3).

In what follows, the graded modules are implicitly assumed to be free, except when explicitly stated.

3.1. Case $\Gamma=\left(\mathbb{Z}_{2}\right)^{n}$. The additive group $\left(\mathbb{Z}_{2}\right)^{n}$ is of finite order $N:=2^{n}$ and we can enumerate its elements following the standard order: the first $q:=2^{n-1}$ elements being the even degrees ordered by lexicographical order, and the last ones being the remaining odd degrees, also ordered lexicographically. E.g. $\left(\mathbb{Z}_{2}\right)^{2}=$ $\{(0,0),(1,1),(0,1),(1,0)\}$. In the following, we denote $\gamma_{i}$ the $i$-th element of $\left(\mathbb{Z}_{2}\right)^{n}$ with respect to this standard order.

This allows to re-order the basis of the considered graded $A$-modules following the degrees of the elements. We call a basis ordered in this way a standard basis. From now on, we only consider this type of basis.

The rank of a free graded module $M$ over a $\left(\mathbb{Z}_{2}\right)^{n}$-commutative algebra is then a $N$-tuple $\mathbf{r}=\left(r_{1}, \ldots, r_{N}\right) \in \mathbb{N}^{N}$ where each $r_{i}$ is the number of basis elements of degree $\gamma_{i}$. Hence, a standard basis $\left\{e_{j}\right\}_{j=1, \ldots, r}$ of a graded $A$-module of rank $\mathbf{r}$ is such that the first $r_{1}$ elements are of degree $\gamma_{1}$, the following $r_{2}$ elements are of degree $\gamma_{2}$, etc.

Consequently, the matrix corresponding to an homogeneous graded morphism $t: M \rightarrow N$ of free $A$-modules of ranks $\mathbf{r}$ and $\mathbf{s}$ respectively, writes as a block matrix

$$
T=\left(\begin{array}{c|c|c}
T_{11} & \ldots & T_{1 N} \\
\hline \ldots & \ldots & \ldots \\
\hline T_{N 1} & \ldots & T_{N N}
\end{array}\right),
$$

where each block $T_{u v}$ (of dimension $s_{u} \times r_{v}$ ) have homogeneous entries of the same degree. This latter is given by $\gamma_{u}+\gamma_{v}+\tilde{t}$, i.e. it depends on both the position $(u, v)$ of the block and the degree of the matrix, which is by definition the degree of the corresponding graded morphism $t$. We will denote by $\mathrm{M}(\mathbf{s}, \mathbf{r} ; A)=$ $\bigoplus_{\gamma \in\left(\mathbb{Z}_{2}\right)^{n}} \mathrm{M}^{\gamma}(\mathbf{s}, \mathbf{r} ; A)$ the space of such matrices, also called graded matrices.

Example 3.1. As a particular case of Clifford algebras, the algebra of quaternions $\mathbb{H}$ is a $\left(\mathbb{Z}_{2}\right)^{3}$-commutative algebra ([10]). We assign to the generators $1, \mathrm{i}, \mathrm{j}$ and $\mathrm{k}$ of $\mathbb{H}$ a degree following the standard order of $\left(\mathbb{Z}_{2}\right)^{3}$, i.e.

$$
\tilde{1}:=(0,0,0), \quad \tilde{\mathrm{i}}:=(0,1,1), \quad \tilde{\mathrm{j}}:=(1,0,1) \quad \text { and } \quad \tilde{\mathrm{k}}:=(1,1,0) .
$$


Note that, as every other Clifford algebra (with the gradation given as in Example 2.1), it is purely even i.e. graded only by the even subgroup of $\left(\mathbb{Z}_{2}\right)^{n}$.

Every graded endomorphism of a free $\mathbb{H}$-module $M$ of rank $\mathbf{r}$ then corresponds to a matrix in $M(\mathbf{r} ; \mathbb{H})$, that is a quaternionic matrix.

The 1-to-1 correspondence

$$
\mathrm{M}^{\gamma}(\mathbf{s}, \mathbf{r} ; A) \simeq \operatorname{Hom}^{\gamma}(M, N),
$$

for every $\gamma \in\left(\mathbb{Z}_{2}\right)^{n}$, permits to transfer the associative graded $A$-algebra structure of $\operatorname{Hom}(M, N)$ to $\mathrm{M}(\mathbf{s}, \mathbf{r} ; A)$. Thus, we get the usual sum and multiplication of matrices, as well as an unusual multiplication of matrices by scalars in $A$ defined as follows.

$$
a T=\left(\begin{array}{c|c|c}
(-1)^{\left\langle\tilde{a}, \gamma_{1}\right\rangle} a T_{11} & \ldots & (-1)^{\left\langle\tilde{a}, \gamma_{1}\right\rangle} a T_{1 N} \\
\hline \ldots & \ldots & \ldots \\
\hline(-1)^{\left\langle\tilde{a}, \gamma_{N}\right\rangle} a T_{N 1} & \ldots & (-1)^{\left\langle\tilde{a}, \gamma_{N}\right\rangle} a T_{N N}
\end{array}\right),
$$

i.e. the $(i, j)$-th entry lying in the $(u, v)$-block is given by

$$
(a T)_{j}^{i}=(-1)^{\left\langle\tilde{a}, \gamma_{u}\right\rangle} a t_{j}^{i} .
$$

Note that the sign appearing here is a direct consequence of (2.4).

In particular, graded endomorphisms $\operatorname{End}(M)$ of a free graded $A$-module $M$ (of finite rank r) can be seen as square graded matrices $\mathrm{M}(\mathbf{r} ; A):=\mathrm{M}(\mathbf{r}, \mathbf{r} ; A)$. With the commutator $(2.5)$ it is another example of $\left(\mathbb{Z}_{2}\right)^{n}$-colored Lie algebra.

3.2. Graded transpose. As in the previous section, let $M$ and $N$ be two graded $A$-modules of ranks $\mathbf{r}$ and $\mathbf{s}$ respectively.

The graded transpose ${ }^{\Gamma \mathrm{t}} T$ of a matrix $T \in \mathrm{M}(\mathbf{s}, \mathbf{r} ; A)$, that corresponds to $t \in \operatorname{Hom}(M, N)$, is defined as the matrix corresponding to the transpose $t^{*} \in$ $\operatorname{Hom}\left(N^{*}, M^{*}\right)$ of $t$. For simplicity, we suppose $t$ to be homogeneous of degree $\tilde{t}$.

We recall that the dual graded $A$-module of $M$ is $M^{*}:=\operatorname{Hom}(M, A)$, so that the dual morphism $t^{*}$ is naturally defined, for all $n^{*} \in N^{*}$ and all $m \in M$, by

$$
\left(t^{*}\left(n^{*}\right), m\right)=(-1)^{\left\langle\tilde{t}, \tilde{n}^{*}\right\rangle}\left(n^{*}, t(m)\right)
$$

where $(-,-)$ denotes the evaluation of the involved morphisms on the corresponding source-module element.

Let $\left\{e_{k}\right\}_{k=1, \ldots, r}$ (resp. $\left\{h_{l}\right\}_{l=1, \ldots, s}$ ) be the basis of $M$ (resp. $N$ ) and let $\left\{\varepsilon^{k}\right\}_{k=1, \ldots, r}$ (resp. $\left\{\eta^{l}\right\}_{l=1, \ldots, s}$ ) the corresponding dual basis. 
Proposition 3.2. The graded transpose ${ }^{\Gamma \mathrm{t}} T$ of a matrix $T=\left(T_{u v}\right) \in \mathrm{M}^{\tilde{t}}(\mathbf{s}, \mathbf{r} ; A)$ (considering here its block form (3.1)) is given by

$$
\left({ }^{\Gamma \mathrm{t}} T\right)_{v u}=(-1)^{\left\langle\gamma_{u}+\gamma_{v}, \tilde{t}+\gamma_{v}\right\rangle \mathrm{t}} T_{u v} .
$$

where $^{\mathrm{t}}$ is the classical transpose.

Proof. From the definition (3.2) of $t^{*}$, we have that

$\left(t^{*}\left(\eta^{j}\right), e_{i}\right)=(-1)^{\left\langle\tilde{t}, \tilde{\left.h_{j}\right\rangle}\right.}\left(\eta^{j}, t\left(e_{i}\right)\right)=(-1)^{\left\langle\tilde{t}, \tilde{h_{j}}\right\rangle} \sum_{k}\left(\eta^{j}, h_{k}\right) t_{i}^{k}=(-1)^{\left\langle\tilde{t}, \tilde{h_{j}}\right\rangle} t_{i}^{j}$.

On the other hand, denoting $t_{i}^{* j}$ the $(i, j)$-entry of the matrix ${ }^{\Gamma \mathrm{t}} T$, we have

$\left(t^{*}\left(\eta^{j}\right), e_{i}\right)=\sum_{k}\left(\varepsilon^{k} t_{k}^{* j}, e_{i}\right)=\sum_{k}(-1)^{\left\langle\left\langle t_{k}^{\tilde{*} j}, \tilde{e}_{i}\right\rangle\right.}\left(\varepsilon^{k}, e_{i}\right) t_{k}^{* j}=(-1)^{\left\langle t_{i}^{* j}, \tilde{e}_{i}\right\rangle} t_{i}^{* j}$

Thus, for all $i, j$,

$$
t_{i}^{* j}=(-1)^{\left\langle t_{i}^{\tilde{j} j}, \tilde{e}_{i}\right\rangle+\left\langle\tilde{t}, \tilde{h_{j}}\right\rangle} t_{i}^{j}
$$

In particular, $t_{i}^{*} j=\tilde{t_{i}^{j}}=\tilde{t}+\tilde{e_{i}}+\tilde{h_{j}}$ for all $i, j$, so that the transpose morphism $t^{*}$ is also homogeneous of same degree $\tilde{t}$. Hence, (3.3) rewrites as

$$
t_{i}^{* j}=(-1)^{\left\langle\tilde{t}+\tilde{e}_{i}, \tilde{h_{j}}+\tilde{e}_{i}\right\rangle} t_{i}^{j}
$$

and the result follows.

In the super case (i.e. $n=1$ ), the graded transpose coincide with the well-known super transpose.

It is easily verified by straightforward computations, that the operation of gradedtransposition satisfy the following familiar property.

Corollary 3.3. For any pair of homogeneous square graded matrices $S, T \in$ $\mathrm{M}(\mathbf{r} ; A)$, of degrees $s$ and $t$ respectively, we have that

$$
{ }^{\Gamma \mathrm{t}}(S T)=(-1)^{\langle s, t\rangle \Gamma \mathrm{t}} T^{\Gamma \mathrm{t}} S .
$$

Consequently, we also have that

$$
\left[{ }^{\Gamma \mathrm{t}} S,{ }^{\Gamma \mathrm{t}} T\right]=-{ }^{\Gamma \mathrm{t}}[S, T] .
$$


3.3. Graded trace. As in the classical context, for any graded $A$-module $M$ there is a natural isomorphism of $A$-modules

$$
M^{*} \otimes_{A} M \simeq \operatorname{End}(M) .
$$

It is given by reading a tensor $\alpha \otimes m \in M^{*} \otimes_{A} M$ as the endomorphism

$$
\alpha \otimes m: \quad M \ni m^{\prime} \mapsto(-1)^{\left\langle\tilde{m}, \tilde{m}^{\prime}\right\rangle} \alpha\left(m^{\prime}\right) m \in M
$$

In the case where $M$ is free (of rank $\mathbf{r}$ ), this endomorphism is represented by a matrix $T=\left(t^{i}{ }_{j}\right) \in \mathrm{M}(\mathbf{r} ; A)$, where the $(i, j)$-th entry is

$$
t^{i}{ }_{j}=(-1)^{\left\langle\tilde{m}, e_{j}\right\rangle+\left\langle\tilde{\alpha_{j}}, \tilde{e}_{j}+\tilde{e}_{i}\right\rangle} \alpha_{j} m^{i}
$$

The above isomorphism permits to define the graded trace of the matrix corresponding to the endomorphism $\alpha \otimes m$ as its contraction $\alpha(m)$ (as a $(1,1)$ tensor).

Definition 3.4. The graded trace of an homogeneous matrix $T=\left(T_{u v}\right) \in \mathrm{M}^{\tilde{t}}(\mathbf{r} ; A)$ (considering here its block form (3.1)) is defined as

$$
\Gamma \operatorname{tr}(T):=\sum_{u}(-1)^{\left\langle\gamma_{u}+\tilde{t}, \gamma_{u}\right\rangle} \operatorname{tr}\left(T_{u u}\right)
$$

where tr denotes the classic trace of a matrix.

It is proved in [6] that $\Gamma \operatorname{tr}: \mathrm{M}(\mathbf{r} ; A) \rightarrow A$ is the unique (up to multiplication by a scalar of degree 0$)$ homomorphism of A-linear colored Lie algebras.

3.4. Graded Berezinian. Fixing a standard basis $\left\{e_{i}\right\}_{i=1, \ldots, r}$ of a free graded $A$ module $M$ permits to represent degree-preserving automorphisms of this module as invertible 0 -degree matrices, the group of which we denote by $\mathrm{GL}^{0}(\mathbf{r} ; A)$. In [6] we have introduced the notion of graded Berezinian for this type of matrices. Let us recall the main result of [6].

There is a unique group homomorphism

$$
\text { ГBer: } \mathrm{GL}^{0}(\mathbf{r} ; A) \rightarrow\left(A^{0}\right)^{\times}
$$

such that:

1) For every block-diagonal matrix $X \in \mathrm{GL}^{0}(\mathbf{r} ; A)$,

$$
\Gamma \operatorname{Ber}(X)=\prod_{u=1}^{q} \operatorname{det}\left(X_{u u}\right) \cdot \prod_{u=q+1}^{N} \operatorname{det}^{-1}\left(X_{u u}\right) .
$$

2) The image of any lower (resp., upper) block-unitriangular matrix in $\mathrm{GL}^{0}(\mathbf{r} ; A)$ equals $1 \in\left(A^{0}\right)^{\times}$. 
Here, $\left(A^{0}\right)^{\times}$denotes the invertible elements of the 0-degree part of $A$, and $q:=$ $N / 2=2^{n-1}$.

Note that, similarly to the classical Berezinian, $\Gamma$ Ber is defined only for 0-degree invertible matrices. In the particular case where the module $M$ is graded by the even part $\left(\mathbb{Z}_{2}\right)_{\overline{0}}^{n}$, the function $\Gamma$ Ber is a polynomial. Moreover, if $A=\mathbb{H}$ then $\Gamma$ Ber coincides with the classical Diedonné determinant (restricted to these type of quaternionic matrices), see [6].

\section{Cohomological definition of the graded Berezinian}

In this section, we define the graded Berezinian module and describe its cohomological interpretation. We obtain the function ГBer from the action of the group of degree-preserving automorphisms. This construction generalizes the one described in [9] for the classical Berezinian.

4.1. Graded Berezinian module. Given a free $A$-module $M$, the corresponding graded Berezinian module $\Gamma \operatorname{Ber}(M)$ is the free $A$-module of total rank 1 built up from formal basis elements $\mathrm{B}\left(\left\{e_{i}\right\}\right)$ for each standard basis $\left\{e_{i}\right\}_{i=1, \ldots, r}$ of $M$. The transformation law induced by a change of basis $e_{i}^{\prime}=e_{j} t_{i}^{j}$ in $M$ (of transition matrix $\left.T=\left(t_{i}^{j}\right)\right)$ is given by

$$
\mathrm{B}\left(\left\{e_{i}^{\prime}\right\}\right)=\mathrm{B}\left(\left\{e_{i}\right\}\right) \Gamma \mathrm{Ber}(T) .
$$

Hence, intuitively, $\Gamma \operatorname{Ber}(M)$ is the free $A$-module of total rank 1 which is functorial with respect to $\mathbf{0}$-degree automorphisms of $A$-modules and if $M$ is concentrated in only one even degree (i.e. is just a classical module) it coincides with the classical determinant module $\operatorname{Det}(M):=\bigwedge^{\text {top }} M$.

The above description of the module $\Gamma \operatorname{Ber}(M)$ is quite abstract. In the text section, we will present an explicit cohomological construction of this module.

4.2. Cohomological construction. Consider the graded-commutative algebra $S_{A}^{\bullet}\left(\Pi M \oplus M^{*}\right)$, where $M$ is a (free) graded $A$-module of rank $\mathbf{r}$.

$S_{A}^{\bullet}\left(\Pi M \oplus M^{*}\right)$ is the $\left(\mathbb{Z}_{2}\right)^{n}$-graded-commutative algebra of polynomials in the graded variables $\Pi e_{i}$ and $\varepsilon^{i}$ with coefficients in $A$. We define the operator $d$ to be left multiplication by the following element of $S_{A}^{2}\left(\Pi M \oplus M^{*}\right)$ :

$$
d=\sum_{i} \Pi e_{i} \varepsilon^{i},
$$

that we also denote by $d$, by abuse of notation. This choice of $d$ is natural since $\left.d\right|_{M} \equiv \Pi$. 
This defines a cochain complex $\mathcal{K}^{\bullet}:=\left(S_{A}^{\bullet}\left(\Pi M \oplus M^{*}\right), d\right)$, thanks to the following result.

Proposition 4.1. The operator $d$ is independent of the choice of the basis and $d^{2}=0$.

Proof. Let us consider a transformation matrix $T=\left(t^{i}{ }_{j}\right) \in \mathrm{GL}^{0}(\mathbf{r} ; A)$ from the basis $\left\{e_{i}\right\}_{i=1, \ldots, r}$ to the basis $\left\{e_{i}^{\prime}\right\}_{i=1, \ldots, r}$ (both standard, $\tilde{e}_{i}^{\prime}=\tilde{e}_{i} \forall i$ ), i.e. $e_{i}^{\prime}=$ $\sum_{j} e_{j} t_{i}^{j}$. The transformation matrix between the induced basis $\left\{\Pi e_{i}\right\}_{i=1, \ldots, r}$ and $\left\{\Pi e_{i}^{\prime}\right\}_{i=1, \ldots, r}$ of $\Pi M$ (resp. between the dual basis $\left\{\varepsilon^{i}\right\}_{i=1, \ldots, r}$ and $\left\{\varepsilon^{\prime i}\right\}_{i=1, \ldots, r}$ of $\left.M^{*}\right)$ is then $T\left(\operatorname{resp} .{ }^{\Gamma t}\left(T^{-1}\right)\right)$, i.e.

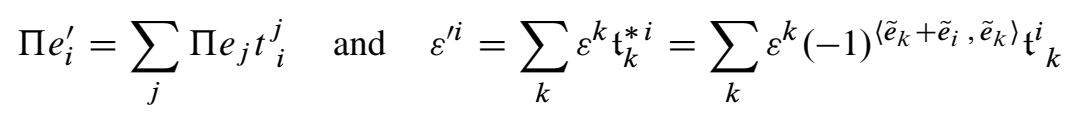

where $\mathrm{t}^{i}{ }_{k}$ denotes the $(i, k)$-th entry of $T^{-1}$.

Hence, we have

$$
\begin{aligned}
\sum_{i} \Pi e_{i}^{\prime} \varepsilon^{\prime i} & =\sum_{i, j, k} \Pi e_{j} t_{i}^{j} \varepsilon^{k}(-1)^{\left\langle\tilde{e}_{i}+\tilde{e}_{k}, \tilde{e}_{k}\right\rangle} \mathfrak{t}^{i}{ }_{k} \\
& =\sum_{j, k} \Pi e_{j} \varepsilon^{k}\left(\sum_{i}(-1)^{\left\langle\tilde{e}_{i}+\tilde{e}_{k}, \tilde{e}_{k}\right\rangle+\left\langle\tilde{e}_{j}+\tilde{e}_{i}, \tilde{e}_{k}\right\rangle} t^{j}{ }_{i} \mathfrak{t}^{i}{ }_{k}\right) \\
& =\sum_{j, k} \Pi e_{j} \varepsilon^{k}(-1)^{\left\langle\tilde{e}_{k}, \tilde{e}_{k}+\tilde{e}_{j}\right\rangle}\left(\sum_{i} t^{j}{ }_{i} \mathfrak{t}^{i}{ }_{k}\right) \\
& =\sum_{j, k} \Pi e_{j} \varepsilon^{k}(-1)^{\left\langle\tilde{e}_{k}, \tilde{e}_{k}+\tilde{e}_{j}\right\rangle} \delta_{k}^{j}=\sum_{j} \Pi e_{j} \varepsilon^{j}
\end{aligned}
$$

so that $d$ is well-defined.

The fact that $d$ squares to zero is easily checked by direct computation, using the graded-commutativity.

To lighten the notation, we will denote by $x_{i}$ the even elements in $\left\{\Pi e_{i}\right\} \cup\left\{\varepsilon^{i}\right\}$ and by $\xi_{i}$ the odd ones (up to sign). More precisely, with $r^{\prime}:=\sum_{i=1}^{q} r_{i}$ indicating the number of basis elements of $M$ of even degree, we set

$x_{i}:=\left\{\begin{array}{ll}\varepsilon^{i} & \text { if } 1 \leq i \leq r^{\prime} \\ \Pi e_{i} & \text { if } r^{\prime}+1 \leq i \leq r\end{array} \quad\right.$ and $\quad \xi_{i}:= \begin{cases}\Pi e_{i} & \text { if } 1 \leq i \leq r^{\prime} \\ -(-1)^{\left\langle\tilde{e}_{i}, \pi\right\rangle} \varepsilon^{i} & \text { if } r^{\prime}+1 \leq i \stackrel{(4.4)}{\leq} r\end{cases}$

By construction, we still have that $\tilde{\xi}_{i}=\tilde{x}_{i}+\pi$ for all $i$.

With this notation, the differential $d$ corresponds to left multiplication by $\sum_{i} \xi_{i} x_{i}$, and $S_{A}^{\bullet}\left(\Pi M \oplus M^{*}\right)$ is now viewed as the $\left(\mathbb{Z}_{2}\right)^{n}$-commutative algebra $A[x, \xi]$ of polynomials in the $\left(\mathbb{Z}_{2}\right)^{n}$-graded variables $x$-s and $\xi$-s. Let us stress the 
fact that the product of polynomials is here the one which is naturally induced by the graded sign rule (2.1).

The elements of the cochain complex $\mathcal{K}^{\bullet}$ at $k$-th level $(k \geq 0)$ are defined as the polynomials in $A[x, \xi]$ with $k$-th total power degree in $\xi$. The element $\xi_{1} \cdots \xi_{r}$ is a cocycle. Indeed, we have that

$$
d\left(\xi_{1} \cdots \xi_{r}\right)=\sum_{i}(-1)^{\left\langle\tilde{\xi}_{i}, \tilde{x}_{i}+\sum_{k<i} \tilde{\xi}_{k}\right\rangle} x_{i} \xi_{1} \cdots \xi_{i-1} \xi_{i}^{2} \xi_{i+1} \cdots \xi_{r}=0
$$

since $\xi_{i}$ are odd and hence squares to 0 . By this same observation, $\mathcal{K}^{k}=0$ for all $k>r$. Hence, this cocycle will play the analogous role of the classical "top element".

\section{Proposition 4.2.}

$$
H^{k}\left(\mathcal{K}^{\bullet}\right)=\left\{\begin{array}{cc}
0 & \text { if } k \neq r \\
{\left[\xi_{1} \cdots \xi_{r}\right] \cdot A} & \text { if } k=r
\end{array}\right.
$$

Proof. Let us consider the operator $\rho=\sum_{i} \frac{\partial}{\partial x_{i}} \frac{\partial}{\partial \xi_{i}}$, where $\frac{\partial}{\partial x_{i}}, \frac{\partial}{\partial \xi_{i}}$ are graded homogeneous partial derivations, i.e.

$$
\begin{aligned}
& \frac{\partial}{\partial x_{i}} x_{j}=\delta_{j}^{i}, \quad \frac{\partial}{\partial x_{i}} \xi_{j}=0 \\
& \frac{\partial}{\partial \xi_{i}} x_{j}=0 \quad, \quad \frac{\partial}{\partial \xi_{i}} \xi_{j}=\delta_{j}^{i} .
\end{aligned}
$$

for all indices $i, j$. Note that the respective degrees are

$$
\frac{\widetilde{\partial}}{\partial x_{i}}=\tilde{x}_{i} \quad \text { and } \quad \frac{\partial}{\partial \xi_{i}}=\tilde{\xi}_{i}=\tilde{x}_{i}+\pi
$$

Let us compute $[\rho, d]$ where $[$,$] is the \left(\mathbb{Z}_{2}\right)^{n}$-commutator (2.5).

$$
\begin{aligned}
{[\rho, d]=} & \sum_{i, j}\left[\frac{\partial}{\partial x_{i}} \frac{\partial}{\partial \xi_{i}}, \xi_{j} x_{j}\right] \\
= & \sum_{i, j} \frac{\partial}{\partial x_{i}}\left[\frac{\partial}{\partial \xi_{i}}, \xi_{j} x_{j}\right]+\sum_{i, j}(-1)^{\left\langle\tilde{x}_{i}+\pi, \pi\right\rangle}\left[\frac{\partial}{\partial x_{i}} \xi_{j} x_{j}\right] \frac{\partial}{\partial \xi_{i}} \\
= & \sum_{i, j} \frac{\partial}{\partial x_{i}}\left[\frac{\partial}{\partial \xi_{i}}, \xi_{j}\right] x_{j}+\sum_{i, j}(-1)^{\left\langle\tilde{x}_{i}+\pi, \tilde{x}_{j}+\pi\right\rangle} \frac{\partial}{\partial x_{i}} \xi_{j}\left[\frac{\partial}{\partial \xi_{i}}, x_{j}\right] \\
& \quad-\sum_{i, j}(-1)^{\left\langle\tilde{x}_{i}, \pi\right\rangle}\left[\frac{\partial}{\partial x_{i}}, \xi_{j}\right] x_{j} \frac{\partial}{\partial \xi_{i}} \\
& \quad-\sum_{i, j}(-1)^{\left\langle\tilde{x}_{i}, \pi\right\rangle+\left\langle\tilde{x}_{i}, \tilde{x}_{j}+\pi\right\rangle} \xi_{j}\left[\frac{\partial}{\partial x_{i}}, x_{j}\right] \frac{\partial}{\partial \xi_{i}}
\end{aligned}
$$


But, by construction,

$$
\begin{array}{lll}
{\left[\frac{\partial}{\partial x_{i}}, x_{j}\right]=\delta_{j}^{i},} & {\left[\frac{\partial}{\partial x_{i}}, \xi_{j}\right]=0,} \\
{\left[\frac{\partial}{\partial \xi_{i}}, x_{j}\right]=0,} & {\left[\frac{\partial}{\partial \xi_{i}}, \xi_{j}\right]=\delta_{j}^{i},}
\end{array}
$$

so that we have

$$
\begin{aligned}
{[\rho, d] } & =\sum_{i} \frac{\partial}{\partial x_{i}} x_{i}-\sum_{i}(-1)^{\left\langle\tilde{x}_{i}, \tilde{x}_{i}\right\rangle} \xi_{i} \frac{\partial}{\partial \xi_{i}} \\
& =\sum_{i}\left(\mathrm{id}+(-1)^{\left\langle\tilde{x}_{i}, \tilde{x}_{i}\right\rangle} x_{i} \frac{\partial}{\partial x_{i}}\right)-\sum_{i}(-1)^{\left\langle\tilde{x}_{i}, \tilde{x}_{i}\right\rangle} \xi_{i} \frac{\partial}{\partial \xi_{i}} \\
& =r \mathrm{id}+\sum_{i} x_{i} \frac{\partial}{\partial x_{i}}-\sum_{i} \xi_{i} \frac{\partial}{\partial \xi_{i}}
\end{aligned}
$$

since each $x_{i}$ have even degree, i.e. $\left\langle\tilde{x}_{i}, \tilde{x}_{i}\right\rangle=0$ for all $i$.

Now, if $P$ is a homogeneous monomial in $\mathcal{K}^{k}$, i.e.

$$
P=\xi^{\alpha} x^{\beta} a_{\alpha \beta}
$$

with $\alpha \in\{0,1\}^{r}$ such that $|\alpha|:=\sum_{i} \alpha_{i}=k, \beta \in \mathbb{N}^{r}$ and $a_{\alpha \beta}$ a homogeneous element of $A$, we have for every $i$ that

$$
x_{i} \frac{\partial}{\partial x_{i}}(P)= \begin{cases}\beta_{i} P & \text { if } \beta_{i} \neq 0 \\ 0 & \text { if } \beta_{i}=0\end{cases}
$$

and

$$
\xi_{i} \frac{\partial}{\partial \xi_{i}}(P)= \begin{cases}P & \text { if } \alpha_{i}=1 \\ 0 & \text { if } \alpha_{i}=0\end{cases}
$$

so that

$$
[\rho, d](P)=(r+|\beta|-k) \operatorname{id}(P) .
$$

In fact, we only have to consider $0 \leq k \leq r$. Hence, $c:=r+|\beta|-k$ is zero if and only if $k=r$ and $\beta=0$. It follows that, for $k \neq r$, we have a cochain homotopy between the identity id and the zero map. It is given by $\rho / c$ on monomials of the same form of $P$. We conclude that $H^{k}\left(\mathcal{K}^{\bullet}\right)=0$ for all $k \neq r$.

It remains to consider the case when $k=r$. By definition,

$$
H^{r}\left(\mathcal{K}^{\bullet}\right)=\operatorname{ker}\left(d: \mathcal{K}^{r} \rightarrow \mathcal{K}^{r+1}\right) / \operatorname{im}\left(d: \mathcal{K}^{r-1} \rightarrow \mathcal{K}^{r}\right)
$$

where $\mathcal{K}^{r}=\left\{\xi_{1} \cdots \xi_{r} Q \mid Q \in A[x]\right\}$ and $\mathcal{K}^{r+1}=0$. Hence, $\operatorname{ker}\left(d: \mathcal{K}^{r} \rightarrow\right.$ $\left.\mathcal{K}^{r+1}\right)=\mathcal{K}^{r}$. On the other hand, by direct computation (e.g. apply $d$ on an element 
of the form $\xi_{\alpha_{1}} \cdots \xi_{\alpha_{r-1}} Q_{\alpha}$ with $Q_{\alpha} \in A[x]$ a homogenous monomial), we obtain $\operatorname{im}\left(d: \mathcal{K}^{r-1} \rightarrow \mathcal{K}^{r}\right)=\xi_{1} \cdots \xi_{r}\left(\sum_{i} A[x] \cdot x_{i}\right)$. In conclusion,

$$
H^{r}\left(\mathcal{K}^{\bullet}\right)=\xi_{1} \cdots \xi_{r} \cdot A[x] / \xi_{1} \cdots \xi_{r}\left(\sum_{i} A[x] \cdot x_{i}\right) \simeq \xi_{1} \cdots \xi_{r} \cdot A
$$

Remark 4.3. a) Proposition 4.2 implies that $H\left(\mathcal{K}^{\bullet}\right)=H^{r}\left(\mathcal{K}^{\bullet}\right) \simeq\left[\xi_{1} \cdots \xi_{r}\right] \cdot A$, hence is a free $A$-module of total rank 1 . The degree of the basis element is

$$
\widetilde{\xi_{1} \cdots \xi_{r}}=\sum_{i=1}^{q}\left(\gamma_{i}+\pi\right) r_{i}+\sum_{i=q+1}^{N} \gamma_{i} r_{i}=r^{\prime} \pi+\sum_{i=1}^{N} \gamma_{i} r_{i}
$$

b) The result is independent of the chosen parity functor $\Pi$ in the cochain complex $\mathcal{K} \bullet$.

A degree-preserving automorphism of $M, \varphi \in \operatorname{Aut}^{0}(M)$ (represented by a matrix $T \in \mathrm{GL}^{0}(\mathbf{r} ; A)$ ), naturally induces two automorphisms

$$
\varphi^{\Pi}: \Pi M \rightarrow \Pi M \quad \text { and } \quad\left(\varphi^{-1}\right)^{*}: M^{*} \rightarrow M^{*},
$$

and hence an automorphism (of $A$-algebras)

$$
\phi: S_{A}^{\bullet}\left(\Pi M \oplus M^{*}\right) \rightarrow S_{A}^{\bullet}\left(\Pi M \oplus M^{*}\right) .
$$

on the "total space" $\mathcal{K}:=\bigoplus_{k} \mathcal{K}^{k}=S_{A}^{\bullet}\left(\Pi M \oplus M^{*}\right)$ of the corresponding complex. Explicitly, it is given by

$$
\phi\left(x_{i}\right)=\left\{\begin{array}{ll}
\left(\varphi^{*}\right)^{-1}\left(x_{i}\right) & 1 \leq i \leq r^{\prime} \\
\varphi^{\Pi}\left(x_{i}\right) & r^{\prime}<i \leq r
\end{array} \quad \text { and } \quad \phi\left(\xi_{i}\right)= \begin{cases}\varphi^{\Pi}\left(\xi_{i}\right) & 1 \leq i \leq r^{\prime} \\
\left(\varphi^{*}\right)^{-1}\left(\xi_{i}\right) & r^{\prime}<i \leq r\end{cases}\right.
$$

i.e. corresponds to matrix multiplication by $T$ on $\Pi M$ and by ${ }^{\Gamma t}\left(T^{-1}\right)$ on $M^{*}$.

The differential $d: \mathcal{K} \rightarrow \mathcal{K}$ is invariant under this transformation (see Proposition 4.1). Hence, we obtain an automorphism on $\operatorname{ker} d / \operatorname{im} d$. This latter module, is equal to

$$
\begin{aligned}
\operatorname{ker} d / \operatorname{im} d=\left(\left.\bigoplus_{k} \operatorname{ker} d\right|_{\mathcal{K}^{k}}\right) /\left(\left.\bigoplus_{k} \operatorname{im} d\right|_{\mathcal{K}^{k-1}}\right) & =\bigoplus_{k}\left(\left.\operatorname{ker} d\right|_{\mathcal{K}^{k}} /\left.\operatorname{im} d\right|_{\mathcal{K}^{k-1}}\right) \\
& =\bigoplus_{k} H^{k}\left(\mathcal{K}^{\bullet}\right)
\end{aligned}
$$

and hence is just $H^{r}\left(\mathcal{K}^{\bullet}\right)$, thanks to Proposition 4.2.

By means of a graded matrix $T \in \mathrm{GL}^{0}(\mathbf{r} ; A)$ representing $\varphi$, the obtained map rewrites as a group action of $\mathrm{GL}^{0}(\mathbf{r} ; A)$ on $H^{r}\left(\mathcal{K}^{\bullet}\right)$. In other words, we have a group morphism

$$
\Phi: \mathrm{GL}^{0}(\mathbf{r} ; A) \rightarrow \operatorname{Aut}^{0}\left(H^{r}\left(\mathcal{K}^{\bullet}\right)\right) \simeq\left(A^{0}\right)^{\times}
$$


given by

$$
\Phi(T)\left(\left[\xi_{1} \cdots \xi_{r}\right]\right)=\left[\phi\left(\xi_{1} \cdots \xi_{r}\right)\right] .
$$

We will now prove that this morphism coincides with the graded Berezinian.

Proposition 4.4. For all $T \in \mathrm{GL}^{0}(\mathbf{r} ; A)$, $\Phi(T)$ is the operator of right multiplication by $\Gamma \operatorname{Ber}(T)$.

Proof. We will first explicit in detail the proof for the super case (i.e. with grading group $\mathbb{Z}_{2}$ ), following the description given in [9]. Hence, in this case the graded Berezinian and the graded trace reduce to the classical Berezinian and the supertrace.

Let us consider two particular types of transformations $\varphi$.

1) Let $\varphi$ be a diagonal transformation, i.e. the corresponding graded matrix is blockdiagonal.

$$
T=\left(\begin{array}{cc}
A & 0 \\
0 & B
\end{array}\right) \in \mathrm{GL}^{0}\left(\mathbf{r}=\left(r^{\prime}, r^{\prime \prime}\right) ; A\right)
$$

The matrix corresponding to the inverse dual is also block-diagonal

$$
{ }^{s t}\left(T^{-1}\right)=\left(\begin{array}{cc}
{ }^{t} A^{-1} & 0 \\
0 & { }^{t} B^{-1}
\end{array}\right) \in \mathrm{GL}^{0}(\mathbf{r} ; A)
$$

Let us denote $a^{i}{ }_{j}$ the entries of $A$ and $\mathfrak{b}_{j}^{i}$ the entries of ${ }^{t} B^{-1}$.

We have that

$$
\phi\left(\xi_{i}\right)= \begin{cases}\varphi^{\Pi}\left(\xi_{i}\right)=\sum_{1 \leq j \leq r^{\prime}} \xi_{j} a_{i}^{j} & \text { for } 1 \leq i \leq r^{\prime} \\ \left(\varphi^{*}\right)^{-1}\left(\xi_{i}\right)=\sum_{r^{\prime}<j \leq r} \xi_{j} \mathfrak{b}_{j-r^{\prime}}^{i-r^{\prime}} & \text { for } r^{\prime}<i \leq r\end{cases}
$$

so that

$$
\begin{aligned}
\phi & \left(\xi_{1} \cdots \xi_{r}\right) \\
& =\varphi^{\Pi}\left(\xi_{1}\right) \cdots \varphi^{\Pi}\left(\xi_{r^{\prime}}\right) \cdot\left(\varphi^{*}\right)^{-1}\left(\xi_{r^{\prime}+1}\right) \cdots\left(\varphi^{*}\right)^{-1}\left(\xi_{r}\right) \\
& =\xi_{1} \cdots \xi_{r}\left(\sum_{\sigma \in \mathfrak{S}_{r^{\prime}}} \operatorname{sign} \sigma a_{1}^{\sigma(1)} \cdots a_{r^{\prime}}^{\sigma\left(r^{\prime}\right)}\right)\left(\sum_{\sigma \in \mathfrak{S}_{r-r^{\prime}}} \operatorname{sign} \sigma \mathfrak{b}_{\sigma(1)}^{1} \cdots \mathfrak{b}_{\sigma\left(r-r^{\prime}\right)}^{r-r^{\prime}}\right) \\
& =\xi_{1} \cdots \xi_{r} \operatorname{det}(A) \operatorname{det}\left(B^{-1}\right) \\
& =\xi_{1} \cdots \xi_{r} \operatorname{Ber}(T) .
\end{aligned}
$$

Here, signs appears because the elements of the subset $\left\{\xi_{i}\right\}_{1 \leq i \leq r^{\prime}}$ (respectively, $\left\{\xi_{i}\right\}_{r^{\prime}<i \leq r}$ ) are of the same odd degree, hence anticommute.

2) Let $\varphi$ be a unitriangular transformation, i.e. the corresponding graded matrix is block-unitriangular. The value of its Berezinian then equals 1 . We will consider 
only the case of an upper unitriangular matrix, the case of a lower unitriangular matrix being similar. Let

$$
T=\left(\begin{array}{cc}
\mathbb{I} & C \\
0 & \mathbb{I}
\end{array}\right) \in \mathrm{GL}^{0}(\mathbf{r} ; A)
$$

Then the corresponding dual inverse is also block-unitriangular, more precisely

$$
{ }^{s t}\left(T^{-1}\right)=\left(\begin{array}{cc}
\mathbb{I} & 0 \\
{ }^{t} C & \mathbb{I}
\end{array}\right)
$$

We have in this case

$$
\phi\left(\xi_{i}\right)= \begin{cases}\varphi^{\Pi}\left(\xi_{i}\right)=\xi_{i} & \text { for } 1 \leq i \leq r^{\prime} \\ \left(\varphi^{*}\right)^{-1}\left(\xi_{i}\right)=\xi_{i} & \text { for } r^{\prime}<i \leq r\end{cases}
$$

so that

$$
\phi\left(\xi_{1} \cdots \xi_{r}\right)=\xi_{1} \cdots \xi_{r}=\xi_{1} \cdots \xi_{r} \operatorname{Ber}(T) .
$$

Hence, we have proved that $\Phi$ coincide with left multiplication by Ber on block diagonal and block unitriangular matrices. By the uniqueness result concerning the Berezinian, this suffices to conclude.

This strategy of proof generalizes to the case of grading group $\left(\mathbb{Z}_{2}\right)^{n}$ for higher $n \in \mathbb{N}$, thanks to the analogous uniqueness result of the graded Berezinian (see Section 3.4). We hence only have to verify 1 . and 2 . in this multigraded case.

Let $M$ be, as usual, a free module of rank $\mathbf{r}=\left(r_{1}, r_{2}, \ldots, r_{N}\right)$. The odd elements $\xi_{i}$ are by construction ordered by degree, so that in each subset $\left\{\xi_{i}\right\}_{\sum_{\alpha<\gamma} r_{\alpha}<i \leq \sum_{\alpha \leq \gamma} r_{\alpha}}$ the elements are of the same odd degree, hence they anticommute. This implies that in the first step the expected signs (and hence the determinants) appears, as in the super case. In the second step, by definition of the graded transpose (see Section 3.2), we still have that if $T$ is a block upper (resp. lower) unitriangular matrix then ${ }^{\Gamma \mathrm{t}}\left(T^{-1}\right)$ is lower (resp. upper) block unitriangular. Let us consider, for simplicity, $n=2$ and $\mathbf{r}=(1,1,1,1)$. We then have only four odd $\xi_{i}$, of two different degrees $(0,1)$ and $(1,0)$. For a graded matrix

$$
T=\left(\begin{array}{cccc}
1 & a & \star & \star \\
& 1 & \star & \star \\
& & 1 & b \\
& & & 1
\end{array}\right) \in \mathrm{GL}^{0}((1,1,1,1) ; A)
$$

we have

$$
\Gamma^{\mathrm{t}}\left(T^{-1}\right)=\left(\begin{array}{cccc}
1 & & & \\
-a & 1 & & \\
\star^{\prime} & \star^{\prime} & 1 & \\
\star^{\prime} & \star^{\prime} & b & 1
\end{array}\right)
$$


We then obtain

$$
\begin{array}{lll}
\varphi^{\Pi}\left(\xi_{1}\right) & =\xi_{1}, & \left(\varphi^{*}\right)^{-1}\left(\xi_{3}\right)=\xi_{3}+\xi_{4} b \\
\varphi^{\Pi}\left(\xi_{2}\right)=\xi_{1} a+\xi_{2}, & \left(\varphi^{*}\right)^{-1}\left(\xi_{4}\right)=\xi_{4},
\end{array}
$$

so that

$$
\phi\left(\xi_{1} \cdots \xi_{4}\right)=\xi_{1}\left(\xi_{1} a+\xi_{2}\right)\left(\xi_{3}+b \xi_{4}\right) \xi_{4}=\xi_{1} \cdots \xi_{4}
$$

since the $\xi_{i}$-s square to zero.

This clearly generalize to arbitrary $\mathbf{r}$ and arbitrary $n$.

We summarize the above statements as follows.

Theorem 4.5. The map

$$
\begin{aligned}
\psi: \Gamma \operatorname{Ber}(M) & \rightarrow H\left(\mathcal{K}^{\bullet}\right) \\
\mathrm{B}\left(\left\{e_{i}\right\}\right) & \mapsto\left[\xi_{1} \cdots \xi_{r}\right]
\end{aligned}
$$

is an A-module isomorphism of degree $r^{\prime} \pi$.

The degree of the isomorphism $\psi$ can be easily understood. Indeed, the $A$ module $\Gamma \operatorname{Ber}(M)$ is then either purely even or purely odd, and this depends only on the parity of $\sum_{i=r^{\prime}+1}^{N} \gamma_{i} r_{i}$. This corresponds, when $n=1$, exactly to the wellknown situation of the classical Berezinian.

\section{Cohomological definition of the graded trace}

As we have seen in the previous section, the assignment $\mathrm{GL}^{0}(\mathbf{r} ; A) \ni T \mapsto$ $\phi_{T} \in \operatorname{Aut}^{0}(\mathcal{K})$, where $\phi_{T}$ is an automorphism of $A$-algebras corresponding to matrix multiplication by $T$ on $\Pi M$ and by ${ }^{\Gamma \mathrm{t}}\left(T^{-1}\right)$ on $M^{*}$, defines a group action for which the differential $d$ is invariant. It induces a group morphism (4.6) which coincides with right multiplication by $\Gamma$ Ber.

In this section, we consider analogously the action of the colored Lie algebra of infinitesimal automorphisms on the complex $\mathcal{K}$. We obtain the graded trace from the action on $\operatorname{Ber}(M)$.

5.1. General construction of the action. Consider, as before, the graded-commutative algebra $\mathcal{K}=S_{A}^{\bullet}\left(\Pi M \oplus M^{*}\right)$, where $M$ is a free $A$-module of rank $\mathbf{r}$ over a graded-commutative algebra $A$. To any homogeneous square matrix $S \in \mathrm{M}(\mathbf{r} ; A)$ of degree $\tilde{S}$ we can associate a graded derivation of the same degree $L_{S} \in \operatorname{Der}^{\tilde{S}}(\mathcal{K})$. $L_{S}$ is given by matrix multiplication by $S$ on $M$ and by matrix multiplication by ${ }_{-}{ }^{\mathrm{t}} \mathrm{S}$ on $M^{*}$. Note that since $\mathcal{K}$ has also an $A$-module structure, it is natural to restrict ourselves only to derivations that are also $A$-module morphisms, i.e.

$$
\operatorname{Der}_{A}(\mathcal{K}):=\{D \in \operatorname{Der}(\mathcal{K}): D(a)=0, \forall a \in A\} \subset \operatorname{End}_{A}(\mathcal{K}) .
$$


Hence, more explicitly, we have an assignment

$$
L: \mathrm{M}(\mathbf{r} ; A) \ni S \mapsto L_{S} \in \operatorname{Der}_{A}(\mathcal{K})
$$

where, for any homogeneous matrix $S, L_{S} \in \operatorname{End}_{A}(\mathcal{K})$ is given on generating elements by

$$
\begin{aligned}
L_{S}\left(\Pi e_{i}\right) & =\sum_{k} \Pi e_{k} s_{i}^{k} \\
\text { and } \quad L_{S}\left(\varepsilon^{i}\right) & =-\sum_{k} \varepsilon^{k}(-1)^{\left\langle\tilde{e}_{i}+\tilde{e}_{k}, \tilde{S}+\tilde{e}_{k}\right\rangle} s_{k}^{i}=-(-1)^{\left\langle\tilde{e}_{i}, \tilde{S}\right\rangle} \sum_{k} s_{k}^{i} \varepsilon^{k}
\end{aligned}
$$

and extends to arbitrary elements by the graded Leibniz rule

$$
L_{S}(a b)=L_{S}(a) b+(-1)^{\langle\tilde{S}, \tilde{a}\rangle} a L_{S}(b),
$$

for all $a, b \in \mathcal{K}$.

In fact, $L$ is a colored Lie algebra morphism of degree 0 . Indeed, we see that the equality

$$
\left[L_{S}, L_{T}\right]=L_{[S, T]}
$$

holds on $M$ by construction, and on $M^{*}$ it follows from (3.4) (Corollary 3.3).

5.2. Deducing the graded trace. The second main result of this paper is as follows.

Theorem 5.1. Given an even matrix $S \in \mathrm{M}_{\overline{0}}(\mathbf{r} ; A)$, its action (by derivation) on the cohomology $H\left(\mathcal{K}^{\bullet}\right)$ is well-defined provided one of the following conditions is satisfied:

1) $S \in \mathrm{M}^{0}(\mathbf{r} ; A)$ and the parity of $\Pi$ is an arbitrary odd element $\pi \in\left(\mathbb{Z}_{2}\right)_{\frac{n}{1}}^{n}$;

2) $S$ is an arbitrary even matrix, $n$ is an odd integer and

$$
\pi=(1,1, \ldots, 1) \in\left(\mathbb{Z}_{2}\right)_{\overline{1}}^{n} .
$$

In both cases, the action of $S$ coincides with the operator of left multiplication by $\Gamma \operatorname{tr}(S)$.

To prove the theorem, let us first determine the conditions sufficient for invariance of the differential $d: \mathcal{K} \rightarrow \mathcal{K}$ under the action (5.1).

Lemma 5.2. $\left[L_{S}, d\right]=0$ if one of the above conditions 1 or 2 is satisfied.

Proof. By definition, the operator $d$ is left multiplication by $\sum_{i} \Pi e_{i} \varepsilon^{i}$. Hence, $\left[L_{S}, d\right]=0$ is equivalent to $L_{S}\left(\sum_{i} \Pi e_{i} \varepsilon^{i}\right)=0$. 
From $(5.1 \mathrm{a}-\mathrm{b})$, we have that

$$
\begin{aligned}
L_{S}\left(\sum_{i} \Pi e_{i} \varepsilon^{i}\right) & =\sum_{i}\left(L_{S}\left(\Pi e_{i}\right) \varepsilon^{i}+(-1)^{\left\langle\tilde{S}, \tilde{e}_{i}+\pi\right\rangle} \Pi e_{i} L_{S}\left(\varepsilon^{i}\right)\right) \\
& =\sum_{i, k} \Pi e_{k} s_{i}^{k} \varepsilon^{i}-(-1)^{\langle\tilde{S}, \pi\rangle} \sum_{i, k} \Pi e_{i} s_{k}^{i} \varepsilon^{k} \\
& =\sum_{u, v} \Pi e_{u} s_{v}^{u} \varepsilon^{v}\left(1-(-1)^{\langle\tilde{S}, \pi\rangle}\right)
\end{aligned}
$$

which is equal to zero if and only if

$$
\langle\tilde{S}, \pi\rangle=0
$$

In particular this holds whenever $\tilde{S}=(0,0, \ldots, 0)$ (then $\pi$ is arbitrary). On the other hand, assuming that $n$ is an odd integer and $\pi=(1,1, \ldots, 1)$, the equality (5.2) then also holds for every homogeneous matrix of even (not necessarily zero) degree.

Consequently, in the two cases of the above proposition, $L$ induces an action (respectively denoted by $\mathcal{L}^{(0)}$ and $\mathcal{L}$ ) on the cohomology $H\left(\mathcal{K}^{\bullet}\right)=H^{r}\left(\mathcal{K}^{\bullet}\right)$. In other words, we have in the first case an algebra morphism

$$
\mathcal{L}^{(0)}: \mathrm{M}^{0}(\mathbf{r} ; A) \rightarrow \operatorname{End}^{0}\left(H^{r}\left(\mathcal{K}^{\bullet}\right)\right) \simeq A^{0}
$$

and in the second case (i.e. when $n$ is odd) an algebra morphism

$$
\mathcal{L}: \mathrm{M}_{\overline{0}}(\mathbf{r} ; A) \rightarrow \operatorname{End}_{\overline{0}}\left(H^{r}\left(\mathcal{K}^{\bullet}\right)\right) \simeq A_{\overline{0}},
$$

both given by

$$
L_{S}\left(\left[\xi_{1} \cdots \xi_{r}\right]\right):=\left[L_{S}\left(\xi_{1} \cdots \xi_{r}\right)\right] .
$$

Here, we use again the $(x, \xi)$ 's notation introduced in (4.4). With this notation, $(5.1 \mathrm{a}-\mathrm{b})$ give in particular

$$
L_{S}\left(\xi_{i}\right)= \begin{cases}\sum_{k=1}^{r^{\prime}} \xi_{k} s_{i}^{k}+\sum_{k=r^{\prime}+1}^{r} x_{k} s_{i}^{k} & \text { if } 1 \leq i \leq r^{\prime} \\ (-1)^{\left\langle\tilde{S}+\pi, \tilde{e}_{i}\right\rangle}\left(\sum_{k=1}^{r^{\prime}} s_{i}^{k} x_{k}-\sum_{k=r^{\prime}+1}^{r}(-1)^{\left\langle\tilde{e}_{k}, \pi\right\rangle} s_{i}^{k} \xi_{k}\right) & \text { if } r^{\prime}<i \leq r\end{cases}
$$


so that

$$
\begin{aligned}
& L_{S}\left(\left[\xi_{1} \cdots \xi_{r}\right]\right)=\left[L_{S}\left(\xi_{1} \cdots \xi_{r}\right)\right] \\
& =\left[\sum_{i=1}^{r}(-1)^{\left\langle\tilde{S}, \sum_{j<i} \tilde{\xi}_{j}\right\rangle} \xi_{1} \cdots \xi_{i-1} L_{S}\left(\xi_{i}\right) \xi_{i+1} \cdots \xi_{r}\right] \\
& =\sum_{i=1}^{r^{\prime}} \sum_{k=1}^{r^{\prime}}(-1)^{\left\langle\tilde{S}, \sum_{j<i} \tilde{\xi}_{j}\right\rangle}\left[\xi_{1} \cdots \xi_{i-1} \xi_{k} s_{i}^{k} \xi_{i+1} \cdots \xi_{r}\right] \\
& +\sum_{i=1}^{r^{\prime}} \sum_{k=r^{\prime}+1}^{r}(-1)^{\left\langle\tilde{S}, \sum_{j<i} \tilde{\xi}_{j}\right\rangle}\left[\xi_{1} \cdots \xi_{i-1} x_{k} s_{i}^{k} \xi_{i+1} \cdots \xi_{r}\right] \\
& +\sum_{i=r^{\prime}+1}^{r} \sum_{k=1}^{r^{\prime}}(-1)^{\left\langle\tilde{S}, \sum_{j<i} \tilde{\xi}_{j}\right\rangle+\left\langle\tilde{S}+\pi, \tilde{e}_{i}\right\rangle}\left[\xi_{1} \cdots \xi_{i-1} s_{k}^{i} x_{k} \xi_{i+1} \cdots \xi_{r}\right] \\
& -\sum_{i=r^{\prime}+1}^{r} \sum_{k=r^{\prime}+1}^{r}(-1)^{\left\langle\tilde{S}, \sum_{j<i} \tilde{\xi}_{j}\right\rangle+\left\langle\tilde{S}+\pi, \tilde{e}_{i}\right\rangle+\left\langle\tilde{e}_{k}, \pi\right\rangle}\left[\xi_{1} \cdots \xi_{i-1} s_{k}^{i} \xi_{k} \xi_{i+1} \cdots \xi_{r}\right] \\
& =\sum_{i=1}^{r^{\prime}}(-1)^{\left\langle\tilde{S}, \sum_{j<i} \tilde{\xi}_{j}\right\rangle}\left[\xi_{1} \cdots \xi_{i-1} \xi_{i} S_{i}{ }_{i} \xi_{i+1} \cdots \xi_{r}\right] \\
& -\sum_{i=r^{\prime}+1}^{r}(-1)^{\left\langle\tilde{S}, \sum_{j<i} \tilde{\xi}_{j}\right\rangle+\left\langle\tilde{S}, \tilde{e}_{i}\right\rangle}\left[\xi_{1} \cdots \xi_{i-1} s_{i}^{i} \xi_{i} \xi_{i+1} \cdots \xi_{r}\right] \\
& =\sum_{i=1}^{r^{\prime}}(-1)^{\left\langle\tilde{S}, \tilde{\xi}_{i}\right\rangle} s_{i}^{i}\left[\xi_{1} \cdots \xi_{r}\right]-\sum_{i=r^{\prime}+1}^{r}(-1)^{\left\langle\tilde{S}, \tilde{e}_{i}\right\rangle} s_{i}^{i}\left[\xi_{1} \cdots \xi_{r}\right] \\
& =\sum_{i=1}^{r^{\prime}}(-1)^{\left\langle\tilde{S}, \tilde{e}_{i}+\pi\right\rangle+\left\langle\tilde{e}_{i}, \tilde{e}_{i}\right\rangle} s_{i}^{i}\left[\xi_{1} \cdots \xi_{r}\right]+\sum_{i=r^{\prime}+1}^{r}(-1)^{\left\langle\tilde{S}, \tilde{e}_{i}\right\rangle+\left\langle\tilde{e}_{i}, \tilde{e}_{i}\right\rangle} s_{i}{ }_{i}\left[\xi_{1} \cdots \xi_{r}\right]
\end{aligned}
$$

Clearly, if we are in one of the two cases described in Theorem 5.1, this rewrites as

$$
L_{S}\left(\left[\xi_{1} \cdots \xi_{r}\right]\right)=\left(\sum_{i=1}^{r}(-1)^{\left\langle\tilde{e}_{i}+\tilde{S}, \tilde{e}_{i}\right\rangle} s_{i}^{i}\right)\left[\xi_{1} \cdots \xi_{r}\right]=\Gamma \operatorname{tr}(S)\left[\xi_{1} \cdots \xi_{r}\right]
$$

Theorem 5.1 is proved.

\section{References}

[1] H. Albuquerque and S. Majid, Quasialgebra structure of the octonions. $J$. Algebra 220 (1999), 188-224. Zbl 0999.17006 MR 1713433 
[2] H. Albuquerque and S. Majid, Clifford algebras obtained by twisting of group algebras. J. Pure Appl. Algebra 171 (2002), 133-148. Zbl 1054.15024 MR 1904473

[3] T. Covolo, J. Grabowski and N. Poncin, $\mathbb{Z}_{2}^{n}$-Supergeometry I: Manifolds and Morphisms, preprint, arXiv:1408.2755, 2014.

[4] T. Covolo, J. Grabowski and N. Poncin, $\mathbb{Z}_{2}^{n}$-Supergeometry II: BatchelorGawedzki Theorem, preprint, arXiv:1408.2939, 2014.

[5] T. Covolo and J.-P. Michel, Determinants over graded-commutative algebras, a categorical viewpoint, preprint, arXiv:1403.7474, 2014.

[6] T. Covolo, V. Ovsienko and N. Poncin, Higher Trace and Berezinian of Matrices over a Clifford Algebra. J. Geom. Phys. 62 (2012), no.11, 2294-2319. Zbl 06086956 MR 2964662

[7] A. W. Knapp, Basic algebra, Volume 1. Springer, 2006. Zbl 1106.00001 MR 2257570

[8] D. Leites (ed.), Seminar on supersymmetry (v. 1. Algebra and Calculus: Main chapters), (J. Bernstein, D. Leites, V. Molotkov, V. Shander). MCCME, Moscow, 2011, 410 pp. (in Russian; a version in English is in preparation but available for perusal).

[9] Yu. Manin, Gauge field theory and complex geometry (2nd ed.). Springer Verlag, Berlin, 1988. Zbl 0641.53001 MR 954833

[10] S. Morier-Genoud, V. Ovsienko, Well, Papa, can you multiply triplets?. Math. Intell. 31 (2009), no.4, 1-2. MR 2562997

[11] S. Morier-Genoud, V. Ovsienko, Simple graded commutative algebras. J. Alg. 323 (2010), no.6, 1649-1664. Zbl 1227.16028 MR 2588129

[12] O. V. Ogievetskii, I. B. Penkov, Serre duality for projective supermanifolds. Funct. Anal. Appl., 18 (1984), no.1, 68-70 (translated from Russian). Zbl 0563.58007 MR 739100

[13] M. Scheunert, Generalized Lie algebras. J. Math. Phys. 20 (1979), no.4, 712 720. Zbl 0423.17003 MR 529734

Received 21 May, 2013

T. Covolo, University of Luxembourg, Mathematics Research Unit, 6, rue Richard Coudenhove-Kalergi, L-1359 Luxembourg, Grand Duchy of Luxembourg

E-mail: tiffany.covolo@uni.lu 